\title{
Factors affecting complications after treatment of epidermal cyst
}

\author{
Man Ki Choi, Kyu Jin Chung \\ Department of Plastic and Reconstructive Surgery, Yeungnam University College of Medicine, Daegu, Korea
}

Received: February 23, 2019

Revised: March 13, 2019

Accepted: March 19, 2019

Corresponding author:

Kyu Jin Chung

Department of Plastic and

Reconstructive Surgery, Yeungnam

University College of Medicine, 170,

Hyeonchung-ro, Nam-gu, Daegu

42415, Korea

Tel: +82-53-620-3480

Fax: +82-53-626-0705

E-mail:guzy7@hanmail.net
Background: Epidermal cysts are the most common benign epithelial tumors in humans. The curative treatment of epidermal cyst is surgical excision. However, only few studies have investigated the cause and mechanism of postoperative complications of epidermal cysts. Therefore, this study aimed to evaluate the factors affecting complications of epidermal cyst after surgical treatment.

Methods: Patients with histologically diagnosed epidermal cysts were selected from among 98 consecutive patients with excised benign cystic tumors from March 2014 to August 2017. Sex, age, size, mobility, site of occurrence, history of infection, history of incision and drainage, complications, history of reoperation, and method of overcoming complications was obtained by analyzing medical records retrospectively.

Results: Five of the 98 patients had wound dehiscence due to surgical infection. Three of them underwent wound healing with conservative treatment without a second operation. The other two patients underwent a second operation and showed signs of preoperative infection. None of the factors showed statistical significance in relation to the occurrence of complications.

Conclusion: Postoperative complications occurred when the excision of the epidermal cyst was performed at preoperative infection sites or at sites with high tension, so attention should be paid to postoperative care.

Keywords: Complications; Epidermal cyst; Surgical wound infection

\section{Introduction}

Epidermal cysts are the most common benign epithelial tumors in humans [1]. Epidermal cysts can occur anywhere in the body, especially on the face (Fig. 1). Epidermal cysts mostly manifest as a mass, but some cysts may appear as granulation tissue with foreign-body granulomatous reaction and chronic inflammation when infection and cyst wall rupture occur [2].

The curative treatment of epidermal cyst is surgical excision [3]. Several treatment methods, including minimal incision surgery, have been reported [4]. However, surgical treatment results in scarring of various lengths. Postoperative complications include infection, bleeding, scarring, and cyst recurrence [2]. In infected epidermal cysts, delayed excision is performed after incision and drainage is performed after infection control (Fig. 2).

However, only few studies have investigated the cause and mechanism of postoperative complications [5]. Therefore, this study aimed to evaluate the factors affecting the post-surgical complications of epidermal cyst treatment. 


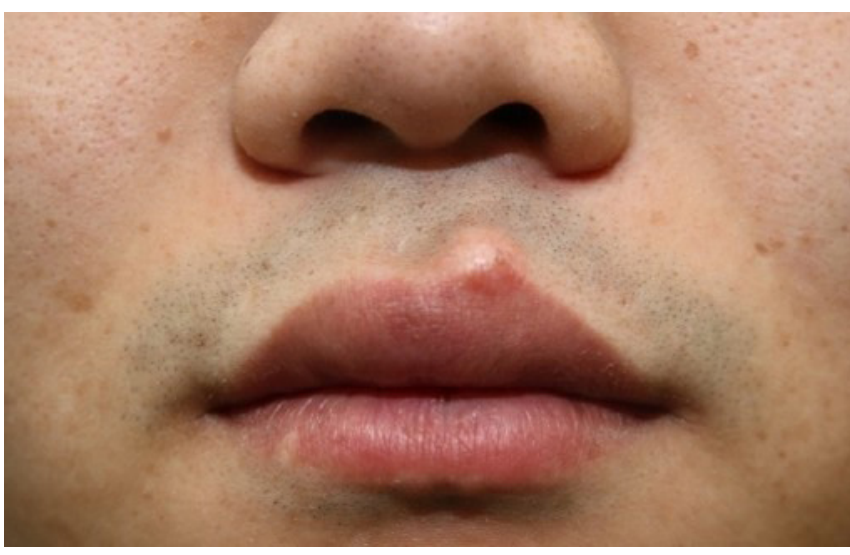

Fig. 1. Epidermal cyst on upper lip.

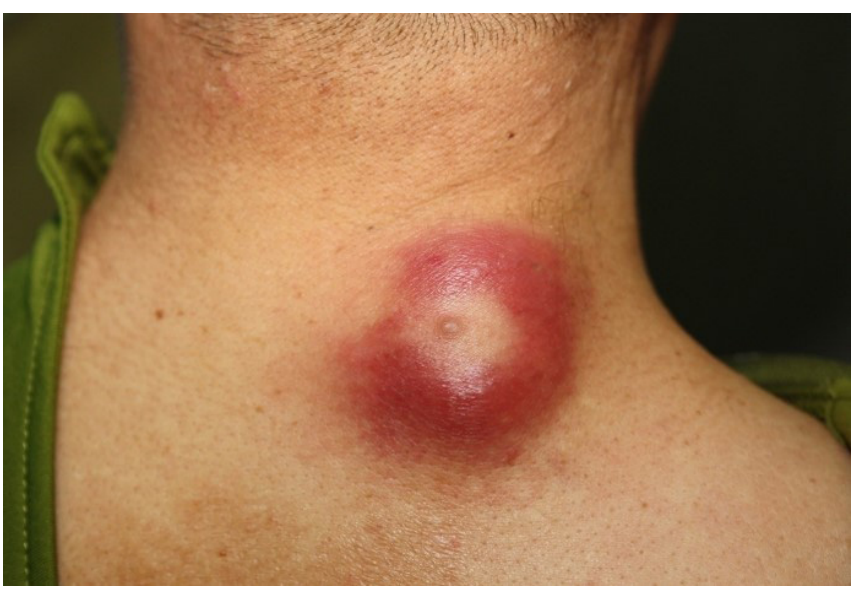

Fig. 2. Infected epidermal cyst.

\section{Materials and methods}

The study was performed in accordance with the principles of the Declaration of Helsinki. Written informed consents were obtained. The present study was approved by the Institutional Review Committee of Yeungnam University hospital (YUMC 2018-06-002-001). The patient provided written informed consent for the publication and the use of his images.

Patients with histologically diagnosed epidermal cysts were selected from among 98 consecutive patients with excised benign cystic tumors from March 2014 to August 2017. Sex, age, size, mobility, site of occurrence, history of infection, history of incision and drainage, complications, history of reoperation, and method of overcoming complications were obtained by analyzing medical records retrospectively. Those with erythema or swelling were considered as having a history of infection. Scarring after surgery was not considered a complication.

If signs of infection, such as erythema or swelling, were observed before the operation, incision and drainage were performed, and the excision was performed after the infection was controlled. After incision and drainage, disinfection treatments such as irrigation were performed daily, and infection was considered to be controlled if discharge or erythema was not observed.

Primary closure was performed after excision in all the patients. Twenty-six patients with preoperative signs of infection were treated with daily irrigation, and excision was performed within 2 weeks.

\section{Surgical procedures}

The skin overlying the epidermal cyst was cleaned with chlorohexidine and physiological saline. Surgery was performed under local anesthetic infiltration of $2 \%$ lidocaine with 1:100,000 epinephrine. The elliptical-shaped incision line was designed to run parallel to the relaxed skin tension line, not crossing over the area of protuberance, and to include the opening of the epidermal cyst. En bloc resection without separating skin and the cyst in the dermal layer was made using a Metzenbaum scissor and electrocauterization. When the cyst wall ruptured during surgery, the suspicious portion of the cyst wall was resected after sufficient irrigation. At the end of the procedure, the wound was inspected to ensure that all of the cyst wall was removed. The face and anterior side of the neck were closed using a nylon 6-0 or 7-0 suture, and the trunk and extremity were closed using a nylon 4-0 or 5-0 suture.

\section{Statistical analysis}

The statistical analysis was performed using IBM SPSS version 24.0 (IBM Co., Armonk, NY, USA). An independent sample $t$ test was used to evaluate the correlation between postoperative complications and age. Correction between the postoperative complications and the size of epidermal cyst were analyzed using the MannWhitney test. The chi-square test was used to evaluate the correlation between the postoperative complications and other factors.

\section{Results}

Of the 98 patients included in the study (Table 1), 74 were men and 24 were women. The mean age was 42.71 years (range, 3-91 years). The mean size was $12 \mathrm{~mm}$ (range, 3-89 mm). Among the 98 cases, 36 had mobility. The distribution of the sites was 48 on the face, 20 on the trunk, 11 on the ear, eight on the neck, eight on the extremity, and three on the scalp. In 26 patients, preoperative signs of infection were observed, and incision and drainage were performed. In 14 patients, cyst wall rupture was observed on 
Table 1. Demographic data of 98 patients with epidermal cyst

\begin{tabular}{lc}
\hline Characteristic & Number (\%) \\
\hline Gender & $74(75.5)$ \\
Male & $24(24.5)$ \\
Female & \\
Mobility & $36(36.7)$ \\
Yes & $62(63.3)$ \\
No & \\
Preoperative infection & $26(26.5)$ \\
Yes & $72(73.5)$ \\
No & \\
Presence of cyst wall rupture & $14(14.3)$ \\
Yes & $84(85.7)$ \\
No & \\
Region & $48(49.0)$ \\
Face & $20(20.4)$ \\
Trunk & $11(11.2)$ \\
Ear & $8(8.2)$ \\
Neck & $8(8.2)$ \\
Extremity & $3(3.0)$ \\
Scalp &
\end{tabular}

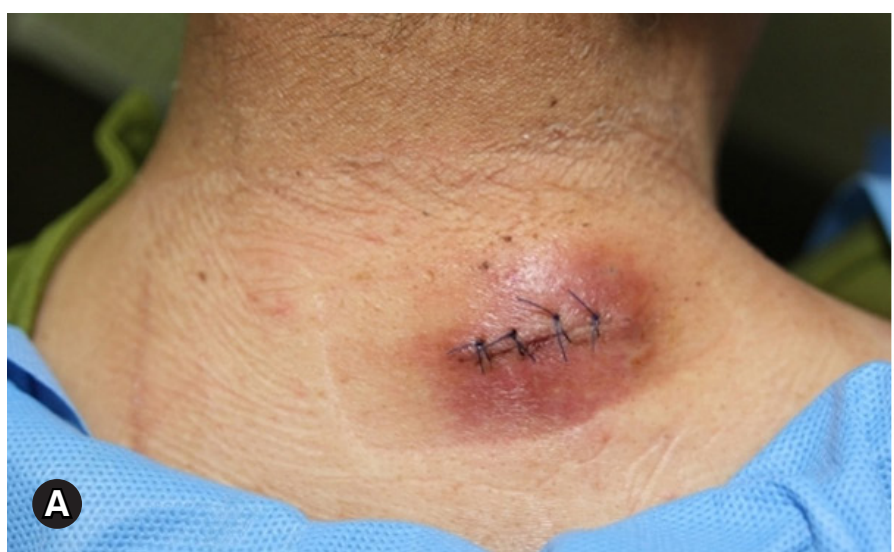

Fig. 3. Wound dehiscence due to postoperative surgical infection. (A) Before stitch out. (B) After stitch out.

histopathological examination. All the patients were diagnosed as having an epidermal cyst based on histopathological examination.

No significant differences were found between the postoperative complications and age $(p=0.322)$. No statistical significance was observed between the postoperative complications and epidermal cyst size $(p=0.267)$. No significant difference was found between postoperative complications and sex $(p=0.093)$, between postoperative complications and preoperative infection

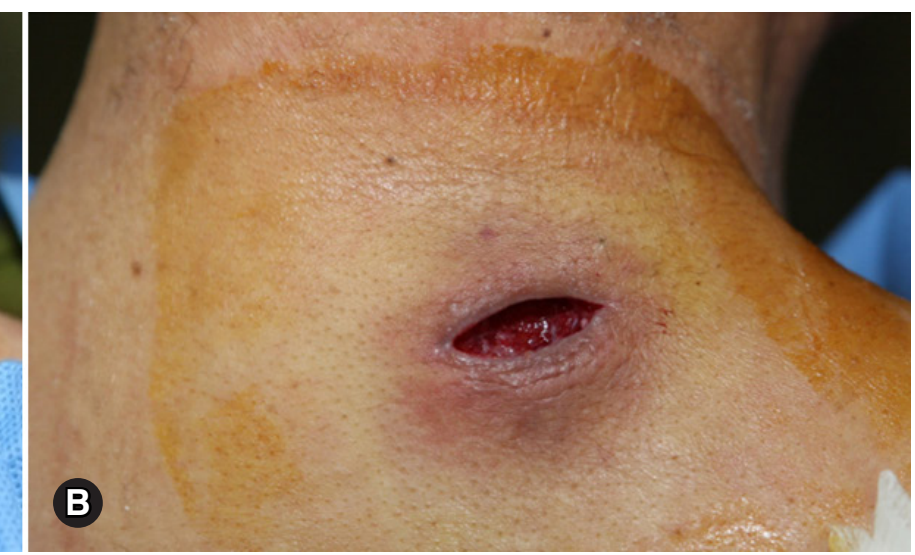

Table 2. Statistical values for comparison between postoperative complications and other factors

\begin{tabular}{lccc}
\hline Characteristic & Complication (-) Complication (+) & $p$-value \\
\hline Gender & 72 & 2 & 0.093 \\
$\quad$ Male & 21 & 3 & \\
$\quad$ Female & 42.3 & 50.4 & 0.322 \\
Age (yr) & 10.8 & 33.7 & 0.267 \\
Size $(\mathrm{mm})$ & & & 0.924 \\
Mobility & 35 & 1 & \\
$\quad$ Yes & 58 & 4 & \\
No & & & 0.606 \\
Preoperative infection & 24 & 2 & \\
$\quad$ Yes & 69 & 3 & \\
No & & & 0.301 \\
Presence of cyst wall & & & \\
rupture & 9 & 5 & \\
Yes & 84 & 0 & \\
$\quad$ No & & & 0.517 \\
Region & 47 & 1 & \\
$\quad$ Face & 18 & 2 & \\
Trunk & 11 & 0 & \\
Ear & 6 & 2 & \\
Neck & 8 & 0 & \\
Extremity & 3 & 0 & \\
$\quad$ Scalp & & & \\
\hline$<0.05$, considered statistically significant. & &
\end{tabular}

$(p=0.606)$, between postoperative complications and cyst wall rupture $(p=0.301)$, between postoperative complications and site of occurrence $(p=0.517)$, and between postoperative complications and mobility $(p=0.924)$ (Table 2$)$.

Surgical site infection was the only complication except scarring. Five patients had wound dehiscence due to surgical site infection (Fig. 3). Five wound sites showed signs of postoperative infection were the back in two, neck in two, and chin in one (Table 3). Three 
Table 3. Five patients with wound dehiscence due to postoperative infection.

\begin{tabular}{lllccc}
\hline Age $(\mathrm{yr})$ & Gender & Site of occurrence & Preoperative infection & Second operation & Presence of cyst wall rupture \\
\hline 63 & Female & Chin & $(-)$ & $(-)$ & $(+)$ \\
62 & Male & Posterior neck & $(+)$ & $(+)$ & $(+)$ \\
45 & Male & Back & $(+)$ & $(+)$ & $(+)$ \\
42 & Female & Posterior neck & $(-)$ & $(-)$ & $(+)$ \\
40 & Female & Back & $(-)$ & $(-)$ & $(+)$ \\
\hline
\end{tabular}
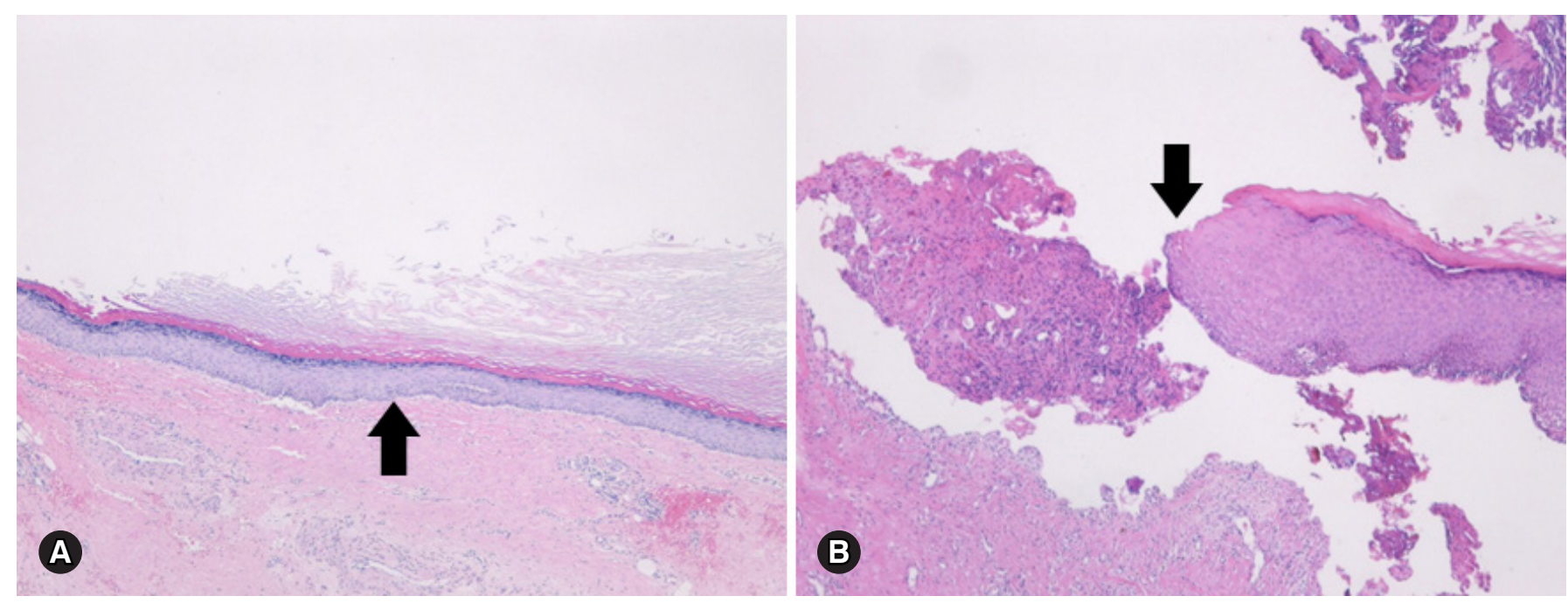

Fig. 4. Histological findings of the epidermal cyst. (A) An arrow shows that the wall of the epidermal cyst is intact (hematoxylin and eosin stain, x40). (B) An arrow shows that the ruptured wall of the epidermal cyst intact (hematoxylin and eosin stain, x100).

of the sites underwent wound healing with conservative treatment without a second operation. They showed no signs of infection before surgery, but the cyst walls were ruptured as shown in the biopsy (Fig. 4). The other two patients underwent a second operation and had signs of preoperative infection. The surgical sites were the posterior neck and back, respectively.

\section{Discussion}

In this study, we investigated the association of various factors and complications that may occur after excision of epidermal cysts. Complications, except for scarring, occurred after excision of the epidermal cyst, which caused wound dehiscence due to infection. Complications occurred in cases with preoperative infection or excised cyst wall rupture. The sites of complications were the back, posterior neck, and chin areas, where tension is relatively high in the body.

Epidermal cysts are smooth, dome-shaped, freely movable, somewhat fluctuant subcutaneous swelling, which are sometimes attached to the skin by a central pore. The cysts are covered with a stratified squamous epithelium that resembles the epidermis or follicular infundibulum.

The first-line treatment of epidermal cyst is simple excision [3]. It is easy to excise if no infection signs are evident. However, in cased with epidermal cyst infection, excision is often difficult because of tissue inflammation or cyst wall rupture. If the cyst wall is not completely removed, the epidermal cyst would recur. Therefore, when an epidermal cyst is infected, surgeons should consider partially incising the cyst and draining it first, and then excising it completely $1-2$ weeks later.

Recently, many studies have shown that epidermal cysts are associated with postoperative scar formation. Minimal incision or laser is used to remove the epidermal cyst with minimal injury to the skin [3]. This helps minimize scar formation after excision of the epidermal cyst. However, only few studies have been conducted on complications after epidermal cyst excision.

In preoperative infection of the epidermal cyst, the cyst wall ruptures and pus or blood in the cyst comes out and infection often follows. The sites of complication after excision of the epidermal cyst are the back, posterior neck, and chin, which have relatively high tension. When an epidermal cyst occurs at the site where a lot of tension is applied, the cyst wall is well ruptured 
owing to an external mechanical force. When the epidermal cyst is excised, the cyst wall could not be easily completely removed if it is ruptured. If the wound is closed while the cyst wall remains, infection may occur and the risk of subsequent recurrence increases.

The limitation of this study is that no statistical significance was attained. None of the factors showed statistical significance in relation to the occurrence of complications. We conclude that the lack of statistical significance was because of the inadequate number of cases in the group with complications. Statistical significance must be confirmed by continuing additional studies.

Postoperative complications occurred when the excision of the epidermal cyst was performed at preoperative infection sites or at sites with high tension. When excision of the epidermal cyst is performed at preoperative infection sites or at sites with high tension, attention should be paid to postoperative care.

\section{Acknowledgements}

This work was supported by a grant from the Chunma medical research foundation, Korea, 2017.

\section{Conflicts of interest}

No potential conflicts of interest relevant to this article was reported.

\section{Patient consent}

Patients provided written consent for the use of their images.

\section{ORCID}

Man Ki Choi, https://orcid.org/0000-0003-2695-4139

Kyu Jin Chung, https://orcid.org/0000-0001-6335-1818

\section{References}

1. Ogawa R. Benign and malignant nonmelanocytic tumors of the skin and soft tissue. In: Neligan PC, Warren RJ, Van Beek AL, editors. 3rd ed. London; New York: Elsevier Saunders; 2013. p. 719-20.

2. Min HJ, Lee JM, Han JK, Kim YJ. Influence factor in thickness of cyst wall of epidermal cysts. J Craniofac Surg 2017;28:e36972.

3. Song SW, Burm JS, Yang WY, Kang SY. Minimally invasive excision of epidermal cysts through a small hole made by a CO2 laser. Arch Plast Surg 2014;41:85-8.

4. Yang HJ, Yang KC. A new method for facial epidermoid cyst removal with minimal incision. J Eur Acad Dermatol Venereol 2009;23:887-90.

5. Turk O, Ozdemir NG, Demirel N, Atci IB, Kanat A, Yolas C. Nontraumatic intradiploic epidermoid cyst and older age: association or causality? J Craniofac Surg 2018;29:e143-6. 